\title{
Analysis of wear abrasion resistance of superhydrophobic acrylonitrile butadiene styrene rubber $(\mathrm{ABS})$ nanocomposites
}

\author{
A. Milionis ${ }^{1, *}$, J. Languasco ${ }^{1}$, E. Loth ${ }^{1}$, I. S. Bayer ${ }^{1,2, *}$ \\ ${ }^{1}$ Department of Mechanical and Aerospace Engineering, University of Virginia, 122 Engineer's Way, \\ 22904 Charlottesville (VA), United States \\ ${ }^{2}$ Smart Materials, Istituto Italiano di Tecnologia, Via Morego 30, 16163 Genoa, Italy
}

\begin{abstract}
Acrylonitrile butadiene styrene rubber (ABS) superhydrophobic nanocomposites containing various concentrations of hydrophobic silica nanoparticles were obtained by solution processing and spray coating on aluminum surfaces. Wear abrasion resistance measurements were conducted on the superhydrophobic nanocomposites as function of silica nanoparticle concentration. A remarkable increase in coating wear abrasion resistance (1700 linear abrasion cycles under $20.5 \mathrm{kPa}$ ) was measured when the nanocomposite coatings were fortified with a synthetic rubber resin adhesive Plasti Dip ${ }^{\mathrm{TM}}$. Detailed wetting measurements before and during abrasion cycles were performed. Changes in surface microtexture were monitored with scanning electron microscopy (SEM) as well as confocal microscopy for surface roughness changes. Wear abrasion of sufficiently thick coatings indicated that there was wear similarity (maintenance of hierarchical texture) throughout the coating thickness during cyclic abrasion. Thermal resistance of the coatings was improved by applying a thin primer layer containing multi-walled carbon nanotubes dispersed in a fluoracrylic polymer solution (Capstone ST-110). The superhydrophobicity of the coatings did not degrade until $420^{\circ} \mathrm{C}$. Simplicity of the process and commercial availability of the materials in large scale can open many practical applications of this coating material system in which water-repellent, wear abrasion resistance is needed particularly for metal surfaces.
\end{abstract}


Keywords: Wear abrasion; Acrylonitrile butadiene styrene rubber; Silica nanoparticles; Superhydrophobic

*Corresponding authors. E-mail addresses: am2vy@virginia.edu (A. Milionis), ilker.bayer@ iit.it (I. S. Bayer).

\section{Introduction}

Practical, repeated and long-term use of large-area, liquid-repellent surfaces requires certain performance criteria such as chemical, physical and thermal stability over time, UV resistance and resistance to external abrasive effects [1-5]. Since many of the superhydrophobic materials are applied in the form of coatings over various surfaces such as metals, plastics, textiles, and stone etc. [5-8], standards developed for organic coatings to test their engineering performance have also been used for superhydrophobic coatings [9-11]. Among them, abrasion resistance is one of the most studied and reported as a measure of liquid repellent surface durability [12-14]. Wear abrasion typically removes a portion of the surface by means of a mechanical contact such as rubbing, sliding back and forth of an object, wind erosion, and so on. It is very important to maintain superhydrophobicity after wear abrasion $[15,16]$ in order to classify such surfaces as "robust" superhydrophobic surfaces. Hereafter, the word "robust" will be used to define superhydrophobic surfaces that can withstand an acceptable degree of wear abrasion. It is worth mentioning that superhydrophobic surfaces often show additional unique properties such as antiicing [17-20]. Therefore, rendering them abrasion resistant is of significant importance for applications that encounter harsh mechanical impacts and require reduced ice adhesion such as aerospace and automotive industry. Since superhydrophobicity requires combination of hierarchical surface texture with hydrophobic chemistry [21-23], the most common strategy for improving and maintaining robustness should involve preservation of micro and nano-scale features associated with the surface topography and the hydrophobic surface chemistry during and after wear abrasion. This is evaluated by performing mechanical abrasion cycles (with constant speed and applied pressure) where the tested superhydrophobic surfaces are abraded by different kinds of abradants that produce different types of wear patterns [24-29].

Two other alternative strategies to maintain liquid repellency under damage include development of self-healing surfaces [30] and easily repairable surfaces [31]. Easily repairable surfaces are materials whose liquid repellent properties can be repaired by simple deposition of a new material over the damaged area. Regarding self-healing repellent surfaces, one approach is based on encapsulation of the liquid repellent component in the pores of rough nano-porous materials. 
When damaged, this component quickly migrates to the damaged surface and recovers its surface properties [32]. The other approach is based on spontaneous self-organization of liquid repellent colloidal particles at interfaces triggered by the action of scratch [33]. In most of the cases, selfhealing process requires a specific amount of time in order to act on the damaged surface. Therefore, under repeated cyclic abrasion, effect and speed of self-healing becomes very critical. Wear abrasion generally causes micron-scale damage and material removal in the form of layers. This can be quite challenging to address in the case of superhydrophobic surfaces which are built upon textured hydrophibic materials such as metals by hydrophobic surface treatments in the form of monolayers (e.g. fluorosilanes) [34]. It is expected that a bulk superhydrophobic nanocomposite with wear similarity property can demonstrate more resilience against wear abrasion. In this case, the underlying exposed surface can maintain a certain degree of roughness along with chemical integrity and can still repel water with the same efficiency under cyclic abrasion conditions [35].

In this work, a facile method is demonstrated to fabricate superhydrophobic surfaces with good wear abrasion resistance as well as thermal stability. A polymer-silica nanocomposite was made by dispersing ABS rubber resin and fumed hydrophobic silica nanoparticles (HMFS) in acetone. In order to enhance coating substrate adhesion, a commercial synthetic rubber solution was also added to the final mixture. The coatings were made by spraying the final solutions on metal plates [36]. The resultant superhydrophobic nanocomposites displayed static water contact angles greater than $150^{\circ}$ and droplet roll-off angles less than $5^{\circ}$. Wear abrasion strongly affected the water droplet roll-off angles such that after 120 abrasion cycles under $20.5 \mathrm{kPa}$, water droplet roll off angles were close to $10^{\circ}$ and after 1700 abrasion cycles under $20.5 \mathrm{kPa}$ water droplet roll off angles reached $45^{\circ}$; however, droplets were still mobile on the surfaces. The static water contact angles, on the other hand, always remained above $150^{\circ}$. To the best of our knowledge, this is among the best wear abrasion resistant superhydrophobic polymer nanocomposite coating developed for metal surfaces reported so far. Note that very robust wear abrasion resistant superhydrophobic textiles have also been made [37-39]. However, in textile technology, the substrates are fiber networks with better pressure dissipation properties and the superhydrophobic treatments are not generally in the form of coatings. 


\section{Materials and methods}

\subsection{Materials}

Acrylonitrile Butadiene Styrene (ABS-P430) resin was purchased from Stratasys, USA in the form of wires. Hydrophobically modified fumed silica (HMFS; Aerosil R-812; particle size 5$50 \mathrm{~nm}$ ) nanoparticles were purchased from Evonik Industries, Germany. Multi-walled carbon nanotubes (MWCNTs), with 110-190 nm diameter and 5-9 $\mu \mathrm{m}$ length, were purchased from Sigma Aldrich, USA. The multi-purpose synthetic rubber Plasti Dip ${ }^{\mathrm{TM}}$ was obtained from Performix, USA and the water-based fluoroacrylic copolymer suspension, Capstone ST-110, was purchased from DuPont, USA. Multipurpose aluminum alloy 6061 plates (1 mm thick) were purchased from McMaster-Carr, USA and were cut into $2.5 \mathrm{~cm}$ wide and $5 \mathrm{~cm}$ long pieces in order to be used as substrates for the coatings. Before the coating application, the substrates were rubbed with P400 grit size sandpaper and cleaned with isopropyl alcohol. All solvents used were of analytical grade.

\subsection{Sample Preparation}

For the preparation of the ABS/HMFS nanocomposites, ABS polymer and HMFS nanoparticles were dissolved and dispersed in acetone in separate vials by $1 \%$ wt. The ABS solution was left overnight until the copolymer completely dissolved and stirred rigorously prior to every use in order to avoid the precipitation of the butadiene rubber particles. The HMFS dispersion was sonicated for 16 min with $42 \mathrm{kHz}$ (Sper Scientific : 100004 - High Powered Ultrasonic Cleaner) in order to improve the dispersion of the particles in acetone. Different solutions were prepared by mixing the ABS and HMFS dispersions in such a way that the concentration of HMFS nanoparticles in ABS (on dry basis) can be adjusted. For the spray coating, a VL double action, internal mix, siphon feed airbrush was used (Paasche, USA). The spray distance from the substrate was held constant at approximately $15 \mathrm{~cm}$ and the air pressure was set at $30 \mathrm{psi}$. A total of $10 \mathrm{ml}$ of the ABS/HMFS dispersions were sprayed on $5 \mathrm{~cm} \times 2.5 \mathrm{~cm}$ aluminum substrates. After spraying, the samples were thermally cured at $240{ }^{\circ} \mathrm{C}$, well above the melting point of ABS $\left(105{ }^{\circ} \mathrm{C}\right)$ resulting in $20 \mu \mathrm{m}$ thick coatings. Thicker coatings $(100 \mu \mathrm{m})$ were also made using higher concentrations of ABS and HMFS in acetone (5 wt \%). These withstood material 
loss due to repeated abrasion better than thinner coatings. A schematic of the spraying process is shown in Fig. 1.

In order to improve substrate adhesion a commercial liquid rubber product (Plasti Dip ${ }^{\mathrm{TM}}$ ) was added in the polymer-nanoparticle dispersions. Plasti $\operatorname{Dip}^{\mathrm{TM}}$ is a moisture-cured, protective, rubber coating that it is specifically designed to adhere to metallic and plastic substrates as a coating. Since its adhesion to both ABS and aluminum is excellent according to the manufacturer specifications, it formed strong bonding between the coating and the aluminum substrate. Moreover, its rubbery nature provides enhanced elasticity to the coating so that it can better dissipate the pressure of abrasion. As a first step for the preparation of the aforementioned threecomponent mixture, the ABS and the HMFS were dissolved and dispersed separately in acetone as described before at a concentration level of $5 \%$ by weight. Afterwards, $25 \mathrm{ml}$ of the $5 \%$ wt. ABS solution was mixed with $10 \mathrm{ml}$ of the 5\% wt. HMFS dispersion. Separately, Plasti Dip ${ }^{\mathrm{TM}}$ liquid product was diluted with equal volume of toluene $(1: 1)$ to reduce its viscosity for spray atomization. Subsequently, $5.25 \mathrm{ml}$ of the diluted Plasti Dip ${ }^{\mathrm{TM}}$ solution was added to the ABS/HMFS dispersion and the final mixture was agitated mechanically. Immediately after stirring, the final mixture was sprayed onto aluminum plates and cured as described above and the samples were ready for abrasion tests. According to the manufacturer Plasti Dip ${ }^{\mathrm{TM}}$ contains $20 \%$ by weight synthetic rubber resin in a mixture of solvents such as Naphta, toluene, hexane and methyl ethyl ketone (MEK). As a result of this mixing process, the final liquid mixture contains approximately equal amounts of ABS resin and Plasti Dip ${ }^{\mathrm{TM}}$ resin. This translates into a dry coating with a polymer matrix made up of 1:1 ABS and synthetic rubber blend.

To enhance thermal resistance characteristics of the coatings in terms of maintaining their superhydrophobicity after exposure to elevated temperatures, a primer layer in the form of a polymer-MWCNT nanocomposite was applied on the aluminum specimens. These nanocomposites were prepared by blending the commercial fluoroacrylic copolymer (Capstone ST-110) and MWCNTs in solution. In particular, $0.3 \mathrm{~g}$ of MWCNTs were added and mixed with $10 \mathrm{ml}$ of Capstone ST-110. Afterwards, an additional $10 \mathrm{ml}$ of toluene and $10 \mathrm{ml}$ of methanol were added to dilute the slurry. The slurry was agitated with a probe sonicator (Sonics VibraCell) for $90 \mathrm{~s}(750 \mathrm{~W}, 20 \mathrm{KHz})$ and it was sprayed from $20 \mathrm{~cm}$ distance for $20 \mathrm{~s}$ time duration with the same airbrush and set pressure. After spray coating onto aluminum plates, the samples 
were left to dry overnight and the final curing treatment was applied at $225^{\circ}$ for $30 \mathrm{~min}$. After curing of this primer layer, the standard ABS/HMFS coatings were spray-coated. This process could be repeated to achieve alternating layers of fluoropolymer-MWCNT and ABS/HMFS nanocomposites.

\subsection{Surface Properties Characterization}

The static apparent water contact angles (APCAs) and droplet roll-off angles (RAs) were measured by a video-based optical contact angle measuring instrument, Ramé-Hart, USA. Deionized water droplets $(10 \mu \mathrm{L})$ were used for the contact angle and roll-off angle measurements as averaged values over three different spots on each sample. For droplet roll-off angles, the substrate was tilted and the substrate tilt angle that the droplet rolled-off from the surface was recorded as RA. All RA values were averaged over three different measurements on each sample. All measurements were performed under ambient conditions. The surface morphology was characterized by SEM, FEI Quanta 650, USA. Surface roughness parameters were calculated by analyzing confocal microscopy images by using a Zeiss 510 Meta System, Germany. Surface roughness values were obtained by using a white light interferometer and then mapping the topography with a built-in 3D reconstruction software.

\subsection{Linear Abrasion}

To quantify the wear abrasion characteristic and the robustness of the coatings, abrasion tests were performed with a linear abrader (Taber Industries, USA) under $20.5 \mathrm{kPa}$ applied pressure. This linear abrasion setup has been used recently in another liquid-repellent coating system but without achieving such performance levels in terms of wear abrasion resistance [40]. This abrasion pressure value is generally above the pressure levels reported in the literature, which appears to be typically close to $10 \mathrm{kPa}$ pressure or less [25,41-44]. Water contact angle and droplet roll-off angle measurements were taken after every 5 linear abrasion cycles. The abradant surface of choice was a piece of crocking cloth and the speed used for the mechanical abrasion was held constant at 15 cycles/min. The abradant during one cycle covered a distance of $5.08 \mathrm{~cm}$ by performing a back and forth linear periodical motion. 


\subsection{Thermal Stability Test}

Thermal stability experiments were performed by gradually heating the specimens on a hot plate until they lost their superhydrophobicity. At every temperature step, the specimens were kept on the heated surface for $10 \mathrm{~min}$ and then allowing the samples to cool down again to ambient conditions for contact angle measurements. After each heating step, the APCA and the RA values were measured and if the samples maintained their superhydrophobic properties, a successive temperature that is $10^{\circ} \mathrm{C}$ higher than the previous was set until degradation was observed in the form of sudden loss of superhydrophobicity and complete surface wetting by water. This point was regarded as the degradation threshold.

\section{Results and discussion}

ABS (acrylonitrile/polybutadiene/styrene) graft copolymers are widely used in many industrial applications including 3D printing. Typically, ABS consists of a styrene/acrylonitrile continuous phase partially grafted to a dispersed butadiene (rubber) phase. The rubber phase acts as an impact modifier, and imparts excellent mechanical properties to the material [45, 46]. It is also a relatively hydrophobic polymer. The water contact angle on a smooth ABS surface is around $85 \pm 3^{\circ}$. However, water adhesion is strong on its surface since water droplets do not roll-off even for $90^{\circ}$ tilt angles. Improving electrical and mechanical properties of ABS by adding nanofillers has been examined by other researchers $[47,48]$. However, superhydrophobic ABS surfaces are very rarely reported. So far, superhydrophobic ABS surfaces have been made by treatment with fluorosilanes [49], not in the form of nanocomposite coatings. It is expected that textured ABS surfaces treated with fluorosilanes may quickly lose their water repellent properties under repeated wear due to abrasion induced texture flattening. In this study, however, the main scope is to exploit impact resistance of $\mathrm{ABS}$ in the form of a nanocomposite material that would ideally maintain its superhydrophobic character even after mechanical damage due to wear abrasion. In the form of nanocomposites containing hydrophobic fumed silica nanoparticles (HMFS) with the right thickness, the coatings can be subjected to repeated wear abrasion with material removal as long as the exposed underlying layers exhibit the same anti-wetting characteristics due to the wear similarity concept [35,50]. Moreover, the hydrophobic nature of ABS remains unchanged due to abrasion, which is considered advantageous [14]. HMFS nanoparticles have an 
extremely low bulk density and high surface area. They are frequently used as thickening agent and viscosity stabilizer in cosmetics, toothpastes, food additives, antiperspirant sprays, medicines, paints, coatings, printing inks, adhesives, etc. Lately, HMFS combined with different organic materials has been used for spray deposition of nanocomposite superhydrophobic coatings for various substrates [51,52]. Their unique agglomeration pattern characteristics in polymer matrices provide the desirable hierarchical morphology comprising both micro- and nano-meter scale roughness typically required for superhydrophobic wetting behavior.

Eleven different nanocomposites were prepared by varying the ratio of ABS and HMFS in increments of $10 \%$ wt. Figure 2 shows some examples of scanning electron micrographs (SEM) of the spray coated nanocomposites before and after the thermal curing corresponding to 0,30 , 60 and $90 \%$ wt. HMFS nanoparticle concentration. Spray atomization of pure polymer solutions can induce surface roughness due to solvent evaporation during atomization [53]. Surface microstructures appear due to spray action and solvent evaporation during atomization of the solutions. This is further enhanced by increasing the HMFS concentration in the polymer as seen in Fig. 2. After the thermal curing step at $240^{\circ} \mathrm{C}$ which is well above the melting point of ABS $\left(105^{\circ} \mathrm{C}\right)$, it can be clearly seen that the surface of pure ABS becomes relatively smooth while in the case of the nanocomposite coatings the surface roughness is still maintained. As such we argue that the melting of ABS matrix served two purposes. The first was to improve substrate adhesion and the second was the better encapsulation of the nanofillers [27,54]. After melting, the surface features were inspected by SEM under higher magnification and nanoscale features due to silica nanoparticles could be identified as shown at the bottom panel of Fig. 2. This combination of micro- and nano-scale roughness along with the hydro-repellent properties of the surface treated silica nanoparticles enables formation of a lotus-leaf like dual-scale topography that leads to superhydrophobic surfaces with low water droplet roll-off angles.

Table 1 shows four relevant surface roughness parameters (average roughness, root mean squared roughness, kurtosis and skewness) before and after the thermal curing step pertaining to the surfaces shown in Fig. 2, namely nanocomposites with HMFS concentrations of 0\%, 30\%, $60 \%$ and $90 \%$ by weight. It can be clearly seen that the average and the root mean squared surface roughness increases by HMFS concentration. After thermal curing of pure ABS, a significant reduction in these two parameters was observed while the other samples maintained their values with some slight variations. The values of kurtosis and skeweness were not 
significantly affected after thermal curing and remained greater than 3 and 0 respectively, indicating presence of peaks and valleys within the surface texture.

All the findings above have a significant impact in the wetting properties of the samples as will be shown below. Figure 3 shows the changes in apparent static water contact angle (APCA) and droplet roll-off angle (RA) as a function of HMFS concentrations in the nanocomposites. It was found that that there was an increasing trend in the values of the APCA and a decreasing trend in the values of RA as HMFS concentration was increased for both before and after thermal curing step. In particular, prior to the thermal curing step, the as sprayed ABS exhibits apparent static water contact angle of $113^{\circ}$ while no RA could be reported since the surfaces are "sticky" and they do not allow the water drops to roll off even at $90^{\circ}$ tilt angles. However, by adding the hydrophobic silica nanoparticles, the coatings were gradually converted to superhydrophobic. Specifically, before the thermal curing step, minimum HMFS concentration necessary for superhydrophobicity was found to be $40 \%$. At this condition the apparent static water contact angle is $160^{\circ}$ and RA is $7^{\circ}$. After this threshold, if the HMFS concentration was further increased, a small gradual increase in APCA and a slight decrease in RA were observed up to 60 $\%$ nanoparticle concentration levels. At this HMFS concentration and above, it was very difficult to maintain water droplets on the surfaces due to the extremely low droplet RAs. Upon superhydrophobic transformation, the APCAs maintain values consistently above $160^{\circ}$. It is worth noting that below $40 \%$ wt. HMFS concentrations the coatings were sticky similar to pure ABS. Thermal curing however, enabled conversion of one of the sticky superhydrophobic nanocomposite (30\% wt. HMFS) into self-cleaning superhydrophobic surface and further significantly reduced the roll-off angle of another nanocomposite with $40 \%$ wt. HMFS concentration, as seen in Fig. 3. The video file in the supporting information demonstrates water droplets bouncing on these extremely water repellent nanocomposite coatings (Video 1).

In order to quantify the wear abrasion characteristics of the superhydrophobic coatings and their surface self-similarity after wear abrasion, a standard abrasion test was performed by using a linear abrader and the wetting properties were measured at different intervals during abrasion cycles. Three different samples were examined for wear abrasion, namely 30, 60 and $90 \%$ wt. HMFS. The total downward abradant pressure was maintained at $20.5 \mathrm{kPa}$, a value relatively high compared to most of the studies that perform such durability tests on superhydrophobic coatings $[16,55,56]$. In Fig. 4a the values of the APCAs and the RAs are plotted against the 
number of abrasion cycles applied on the superhydrophobic nanocomposites. It is evident that the durability of the coatings is influenced by the HMFS concentration. In particular, samples containing $30 \%$ wt. HMFS were found to be the most durable and they were able to maintain their superhydrophobic properties (APCA $>150^{\circ}$ and $\mathrm{RA}<10^{\circ}$ ) for 25 abrasion cycles. On the other hand, nanocomposites with 60\% and $90 \%$ HMFS concentrations were able to withstand 15 and 4 abrasion cycles, respectively. It is therefore clear that ABS matrix significantly increases the durability performance and nanocomposites with $70 \%$ wt. ABS and 30\% wt. HMFS were found to have the best compromise between wear abrasion resistance and superhydrophobicity as seen the graphs of Fig.3 and Fig. 4a. It is worth noting that the coatings exhibit a wearsimilarity behavior in their surface micro-structure after wear abrasion which proves that the dispersion of the HMFS is homogeneous throughout the entire coating thickness. Loss of wearsimilarity in surface texture results in a "sticky wetting state" after the critical number of abrasion cycles. For instance, on these surfaces, the water drops were pinned even at $90^{\circ}$ tilt angles after 25 wear abrasion cycles. This sudden change in the dynamic behavior of the water drops is attributed to the formation of several "pinning sites" after the 25 abrasion cycles. Inspection of SEM images taken on such surfaces as shown in Figs. 4b (low magnification) and Fig. 4c (high magnification) indicates that some areas can be identified where the coating had been removed after 25 abrasion cycles (notice the exposed aluminum substrate in the images). Since the aluminum substrate is hydrophilic, these exposed sites cause the drops to get "pinned" and eventually a Cassie-Baxter to Wenzel transition takes place. However, the fact that there was no gradual degradation of the wetting properties but rather a sudden appearance of a "sticky state" immediately after 25 abrasion cycles indicates that the abraded surface should maintain some sort of wear similarity such that the subsequent underlying surface contain similar surface features that tend to be close to the as sprayed one.

This performance is considered fairly satisfactory for a superhydrophobic nanocomposite in terms of mechanical durability. However it was possible to significantly improve it by almost an order of magnitude (in terms of number of abrasion cycles) by adding a third component in the nanocomposite mixture. For this purpose, a commercial rubber product was introduced in the dispersion as described earlier in the Experimental section in detail. Plasti Dip ${ }^{\mathrm{TM}}$ is a synthetic rubber product and has very good adhesion to both aluminum and ABS surfaces. It is hydrophobic (approximately $100^{\circ}$ APCA) while at the same time quite elastic and thus can 
ideally absorb some part of the friction force during mechanical contact. As shown in Fig. 5a, there is a slight increase in the values of water droplet roll-off angles compared to nanocomposites containing no Plasti Dip ${ }^{\mathrm{TM}}$. However, as seen in the same figure, addition of Plasti Dip ${ }^{\mathrm{TM}}$ results in preservation of superhydrophobicity for up to 120 cycles (with RAs $<10^{\circ}$ ). Moreover, if wear abrasion cycles are continued further, the nanocomposites prove to be very resilient towards losing their water repellency for up to 1700 cycles of linear abrasion. Namely, the coatings are able to maintain APCAs greater than $150^{\circ}$ while RAs, although higher than $10^{\circ}$, remain less than $40^{\circ}$, which is a Teflon-like behavior, meaning that the droplets still roll away from the surfaces after 1700 wear abrasion cycles. This finding, to the best of our knowledge, is among the best performances that have ever been reported for a superhydrophobic nanocomposite coating on metals. In this case no sudden Cassie-Baxter to Wenzel transition took place since the rubber was adhered to the aluminum substrate very well and no exposed aluminum sites appeared after 1700 cycles of linear abrasion as it can be seen in the SEM images before and after abrasion (Figs. 5 b,c). Therefore, the loss of superhydrophobicity in this case, is attributed to the gradual wearing away or loss of the HMFS particles during increasing abrasion cycles. It is worth noting that these nanocomposite coatings can also be applied on soft elastic surfaces such as rubber films (Fig. 5d). The second video file in the supporting information demonstrates a free standing rubber film coated with the thermally cured nanocomposite rubber/ABS/HMFS mixture. As seen, the coatings maintain their water repellency even after rubbing them intensively (Video 2). The third video file in the supporting information demonstrates that these superhydrophobic films are also very flexible and they can maintain their water repellency even after severe deformation (Video 3).

An attempt was also made to enhance the thermal stability [3,57-62] of these wear abrasion resistant coatings. By thermal stability we mean the ability of the nanocomposites to maintain their water repellency after being exposed to temperatures in excess of $400^{\circ} \mathrm{C}$. Note that many low surface energy polymers and fluorinated silanes used for superhydrophobic functionalization cannot easily resist such temperature levels. To achieve this, a primer layer containing a mixture of fluoroacrylic polymer-MWCNTs dispersion was applied that tends to dissipate the heat from the substrate into the coating, thus maintaining superhydrophobicity at elevated temperatures. The main reason for using the fluoroacrylic polymer-MWCNT nanocomposite was the fact that our earlier studies indicated that the fluoroacrylic polymer (Capstone ST-100 or ST-110) has a 
good degree of compatibility with MWCNTs or carbon nanofibers [54], in the sense that asreceived MWCNTs do not need chemical wall functionalization for dispersing in solutions containing the fluoropolymer if the solution preparation is achieved with the use of proper solvents [54]. At the same time the polymer itself has good adhesive properties to polar surfaces such as glass, metals and ceramics $[9,35]$. As a result of the carbon nanotube containing primer layer, the superhydrophobic ABS nanocomposites lost their superhydrophobicity at $420^{\circ} \mathrm{C}$ instead of $370^{\circ}$ without the primer layer.

Note that HMFS nanoparticles lose their hydrophobic surface functionalization at temperatures greater than $300^{\circ} \mathrm{C}$ as indicated by the manufacturer technical specs. Carbon based materials are known to exhibit good thermal conductivity $[63,64]$. Therefore, it was anticipated that applying a nanocomposite coating containing MWCNTs prior to the application of the ABS/HMFS nanocomposite, it could be possible to dissipate some of the heat transmitted from the aluminum substrate and therefore expand the lifetime of the superhydrophobic coatings to higher temperature regimes. As a result, another superhydrophobic coating comprising fluoroacrylic/MWCNTs was applied as a primer or a sandwich layer between two ABS/HMFS layers and its effect on the thermal durability of the superhydrophobicity was examined. A control sample was also made for comparison. In Fig. 6, a sketch is shown depicting the three different samples tested. Thermal treatment in the form of increasing temperatures was performed as described in the Experimental section and the wetting properties after each step were evaluated. The control sample lost its superhydrophobicity at temperatures close $370^{\circ} \mathrm{C}$ while the sample with the fluoroacrylic/MWCNTs nanocomposite as primer and as sandwiched layer showed significantly better thermal stability such that superhydrophobicity was maintained until $410^{\circ} \mathrm{C}$ and $420^{\circ} \mathrm{C}$, respectively. This indicated that fluoroacrylic/MWCNTs nanocomposites as primer layer or as a sandwich layer could expand superhydrophobic thermal life by $40-50{ }^{\circ} \mathrm{C}$. Figure 6 shows photographs of the superhydrophilic coatings, after respective temperature thresholds were reached and the superhydrophobicity was lost. The circular traces seen in the photographs are due to completely spread water drops.

\section{Conclusions}

In conclusion, wear abrasion resistant acrylonitrile butadiene styrene rubber (ABS) superhydrophobic nanocomposite coatings containing hydrophobic silica nanoparticles were 
developed on aluminum surfaces. Wear abrasion resistance under $20.5 \mathrm{kPa}$ increased tremendously (up to 1700 cycles) when the coating formulations were fortified with a synthetic rubber resin adhesive, Plasti Dip ${ }^{\mathrm{TM}}$. Wear abrasion of sufficiently thick coatings demonstrated wear similarity (maintenance of hierarchical texture) throughout the coating thickness during cyclic abrasion. Thermal resistance of the coatings was improved by applying a thin primer layer containing multi-walled carbon nanotubes. Superhydrophobicity of the coatings did not degrade until $420^{\circ} \mathrm{C}$. Due to the simplicity and efficiency of this technique such coatings can find a wide range of industrial and engineering applications such as in vehicles, treatment of construction materials, motors, wind turbines, etc.

\section{Acknowledgements}

The authors would like to thank Mr. Khanh Dang and Mr. Carlos Noyes from the Department of Mechanical and Aerospace Engineering of the University of Virginia for their contribution in the experimental work. The authors would also like to acknowledge financial support from the National Science Foundation.

\section{References}

[1] T. Ishizaki, N. Saito, Rapid formation of a superhydrophobic surface on a magnesium alloy coated with a cerium oxide film by a simple immersion process at room temperature and its chemical stability. Langmuir 26 (2010) 9749-9755, DOI: 10.1021/la100474x.

[2] L. Zhai, F.C. Cebeci, R.E. Cohen, M.F. Rubner, Stable superhydrophobic coatings from polyelectrolyte multilayers. Nano Lett. 4 (2004) 1349-1353, DOI: 10.1021/nl049463j.

[3] X. Deng, L. Mammen, Y. Zhao, P. Lellig, K. Mullen, C. Li, H. Butt, D. Vollmer, Transparent, thermally stable and mechanically durable robust superhydrophobic surfaces made from porous silica capsules. Adv. Mater. 23 (2011) 2962-2965, DOI: 10.1002/adma.201100410.

[4] T.I. Isimyan, T. Wang, S. Rohani, A novel method to prepare superhydrophobic, UV resistance and anticorrosion steel surface. Chem Eng. J. 210 (2012) 182-187. doi:10.1016/j.cej.2012.08.090.

[5] D. Ebert, B. Bhushan, Transparent, superhydrophobic, and wear-resistant coatings on glass and polymer substrates using $\mathrm{SiO}_{2}, \mathrm{ZnO}$, and ITO nanoparticles. Langmuir 28 (2012) 11391-11399, DOI: 10.1021/la301479c.

[6] A. Milionis, KG. Krishnan, E. Loth, Hemolymph drop impact outcomes on surfaces with varying wettability. Appl. Surf. Sci. 345 (2015) 36-43, doi:10.1016/j.apsusc.2015.02.185.

[7] J. Zimmermann, F.A. Reifler, G. Fortunato, L. Gerhardt, S. Seeger, A simple, one-step approach to durable and robust superhydrophobic textiles. Adv. Funct. Mater. 18 (2008) 3662-3669. 
[8] P.N. Manoudis, A. Tsakalof, I. Karapanagiotis, I. Zuburtikudis, C. Panayiotou, Fabrication of superhydrophobic surfaces for enhanced stone protection. Surf. Coat. Tech. 203 (2009) 1322-1328.

[9] A. Steele, I. Bayer, E. Loth, Adhesion strength and superhydrophobicity of polyurethane/organoclay nanocomposite coatings. J. Appl. Polym. Sci. 125 (2012) E445-E452, DOI: 10.1002/app.36312.

[10]X. Zhang, F. Shi, J. Niu, Y. Jiang, Z. Wang, Superhydrophobic surfaces: from structural control to functional application. J. Mater. Chem. 18 (2008) 621-633, DOI: 10.1039/B711226B.

[11] J. Zimmermann, F.A. Reifler, U. Schrade, G.R.J. Artus, S. Seeger, Long term environmental durability of a superhydrophobic silicone nanofilament coating. Colloid. Surface. A 302 (2007) 234-240. doi:10.1016/j.colsurfa.2007.02.033.

[12]C. Su, Y. Xu, F. Gong, F. Wang, C. Li, The abrasion resistance of a superhydrophobic surface comprised of polyurethane elastomer. Soft Matter 6 (2010) 6068-6071. DOI: 10.1039/C0SM00804D.

[13] Q.F. Xu, B. Mondal, A. Lyons, Fabricating superhydrophobic polymer surfaces with excellent abrasion resistance by a simple lamination templating method. ACS Appl. Mater. Interfaces 3 (2011) 3508-3514, DOI: 10.1021/am200741f.

[14]T. Verho, C. Bower, P. Andrew, S. Franssila, O. Ikkala, R.H.A. Ras, Mechanically Durable Superhydrophobic Surfaces. Adv. Mater. 23 (2011) 673-678, DOI: 10.1002/adma.201003129.

[15]H. Zhou, H. Wang, H. Niu, A. Gestos, X. Wang, T. Lin, Fluoroalkyl silane modified silicone rubber/nanoparticle composite: a super durable, robust superhydrophobic fabric coating. Adv. Mater. 24 (2012) 2409-2412, DOI: 10.1002/adma.201200184.

[16]X. Zhu, Z. Zhang, X. Men, J. Yang, K. Wang, X. Xu, X. Zhou, Q. Xue, Robust superhydrophobic surfaces with mechanical durability and easy repairability. J. Mater. Chem. 21 (2011) 15793-15797, DOI: 10.1039/C1JM12513C.

[17]A. Davis, Y.H. Yeong, A. Steele, I.S. Bayer, E. Loth. Superhydrophobic nanocomposite surface topography and ice adhesion. ACS Appl. Mater. Interfaces 6 (2014) 9272-9279, DOI: 10.1021/am501640h.

[18] Y. Wang, J. Xue, Q. Wang, Q. Chen, J. Ding. Verification of icephobic/anti-icing properties of a superhydrophobic surface. ACS Appl. Mater. Interfaces 5 (2013) 3370-3381, DOI: 10.1021/am400429q.

[19]L.B. Boinovich, A.M. Emelyanenko, V.K. Ivanov, A.S. Pashinin. Durable icephobic coatings for stainless steel. ACS Appl. Mater. Interfaces 5 (2013) 2549-2554, DOI: 10.1021/am3031272.

[20] S. Farhadi, M. Farzaneh, S.A. Kulinich. Anti-icing performance of superhydrophobic surfaces. Appl. Surf. Sci. 257 (2011) 6264-6269, doi:10.1016/j.apsusc.2011.02.057.

[21] A. Milionis, D. Fragouli, L. Martiradonna, G.C. Anyfantis, P.D. Cozzoli, I.S. Bayer, A. Athanassiou, Spatially controlled surface energy traps on superhydrophobic surfaces. ACS Appl. Mater. Interfaces 6 (2014) 1036-1043, DOI: 10.1021/am404565a.

[22] A. Milionis, L. Martiradonna, G.C. Anyfantis, P.D. Cozzoli, I.S. Bayer, D. Fragouli, A. Athanassiou, Control of the water adhesion on hydrophobic micropillars by spray coating technique. Colloid Polym. Sci. 291 (2013) 401-407, DOI 10.1007/s00396-012-2752-5.

[23] A. Milionis, R. Giannuzzi, I.S. Bayer, E.L. Papadopoulou, R. Ruffilli, M. Manca, A. Athanassiou. Selfcleaning organic/inorganic photo-sensors. ACS Appl. Mater. Interfaces 5 (2013) 7139-7145. DOI: 10.1021/am401476k. 
[24]L. Xiong, L.L. Kendrick, H. Heusser, J.C. Webb, B.J. Sparks, J.T. Goetz, W. Guo, C.M. Stafford, M.D. Blanton, S. Nazarenko, D.L. Patton, Spray-deposition and photopolymerization of organic-inorganic thiol-ene resins for fabrication of superamphiphobic surfaces. ACS Appl. Mater. Interfaces 6 (2014) 10763-10774, doi: 10.1021/am502691g.

[25] Y. Zhang, D. Ge, S. Yang, Spray-coating of superhydrophobic aluminum alloys with enhanced mechanical robustness. J. Colloid Interf. Sci. 423 (2014) 101-107, doi: 10.1016/j.jcis.2014.02.024.

[26] A. Steele, B.N. Nayak, A. Davis, M.C. Gupta, E. Loth, Linear abrasion of a titanium superhydrophobic surface prepared by ultrafast laser microtexturing. J. Micromech. Microeng. 23 (2013) 115012, doi:10.1088/0960-1317/23/11/115012.

[27]A. Milionis, R. Ruffilli, I.S. Bayer, Superhydrophobic nanocomposites from biodegradable thermoplastic starch composites (Mater-Bi®), hydrophobic nano-silica and lycopodium spores. RSC Adv. 4 (2014) 34395-34404, DOI: 10.1039/C4RA04117H.

[28]J. Wu, J. Zhang, B. Li, L. Fan, L. Li, A. Wang, Facile preparation of super durable superhydrophobic materials. J. Colloid Interf. Sci. 432 (2014) 31-42, doi: 10.1016/j.jcis.2014.06.046.

[29] S. Barthwal, Y.S. Kim, S. Lim, Mechanically robust superamniphobic aluminum surface with nanoporeembedded microtexture. Langmuir 29 (2013) 11966-11974, DOI: 10.1021/la402600h.

[30]L. Ionov, A. Synytska, Self-healing superhydrophobic materials. Phys. Chem. Chem. Phys. 14 (2012) 10497-10502, DOI: 10.1039/C2CP41377A.

[31] J. Li, X. Liu, Y. Ye, H. Zhou, J. Chen, A simple solution-immersion process for the fabrication of superhydrophobic cupric stearate surface with easy repairable property. Appl. Surf. Sci. 258 (2011) 17721775, doi:10.1016/j.apsusc.2011.10.042.

[32] Y. Li, L. Li, J. Sun, Bioinspired self-healing superhydrophobic coatings. Angew. Chem. Int. Ed. 49 (2010) 6129-6133, DOI: 10.1002/anie.201001258.

[33]N. Puretskiy, G. Stoychev, M. Stamm, L. Ionov. Switchable surfaces based on freely floating colloidal particles. ACS Appl. Mater. Interfaces, 2 (2010) 2944-2948, DOI: 10.1021/am100634m.

[34] Y. Xiu, Y. Liu, D.W. Hess, C.P. Wong, Mechanically robust superhydrophobicity on hierarchically structured Si surfaces. Nanotechnology 21 (2010) doi:10.1088/0957-4484/21/15/155705.

[35]I.S. Bayer, A. Brown, A. Steele, E. Loth, Transforming anaerobic adhesives into highly durable and abrasion resistant superhydrophobic organoclay nanocomposite films: a new hybrid spray adhesive for tough superhydrophobicity. Appl. Phys. Express 2 (2009) 125003, doi:10.1143/APEX.2.125003.

[36] T. Schutzius, I.S. Bayer, J. Qin, D. Waldroup, C.M. Megaridis, Water-based, nonfluorinated dispersions for environmentally benign, large-area, superhydrophobic coatings. ACS Appl. Mater. Interfaces 5 (2013) 13419-13425, DOI: 10.1021/am4043307.

[37]J. Wu, J. Li, B. Deng, H. Jiang, Z. Wang, M. Yu, L. Li, C. Xing, Y. Li, Self-healing of the superhydrophobicity by ironing for the abrasion durable superhydrophobic cotton fabrics. Sci. Rep. 3 (2013) 2951, doi:10.1038/srep02951.

[38]H. Wang, H. Zhou, A. Gestos, J. Fang, H. Niu, J. Ding, T. Lin, Robust, electro-conductive, self-healing superamphiiphobic fabric prepared by one-step vapour-phase polymerisation of poly $(3,4-$ 
ethylenedioxythiophene) in the presence of fluorinated decyl polyhedral oligomeric silsesquioxane and fluorinated alkyl silane. Soft Matter 9 (2013) 277-282, DOI: 10.1039/c2sm26871j.

[39] H. Zhou, H. Wang, H. Niu, A. Gestos, T. Lin, Robust, self-healing superamphiphobic fabrics prepared by two-step coating of fluoro-containing polymer, fluoroalkyl silane, and modified silica nanoparticles. Adv. Funct. Mater. 23 (2013) 1664-1670, DOI: 10.1002/adfm.201202030.

[40] A. Milionis, K. Dang, M. Prato, E. Loth, I.S. Bayer. Liquid repellent nanocomposites obtained from onestep water-based spray. J. Mater. Chem. A (2015), DOI: 10.1039/C5TA02672E.

[41]K. Chen, S. Zhou and L. Wu. Facile fabrication of self-repairing superhydrophobic coatings. Chem. Commun. 50 (2014) 11891-11894, DOI: 10.1039/c3cc49251f.

[42]H. Zhou, S. Lin, Y. Tu, G. Liu, J. Hu, F. Li, L. Miao, G. Zhang, H. Luo, F. Liu, C. Hou, M. Hu. Simple approach towards fabrication of highly durable and robust superhydrophobic cotton fabric from functional diblock copolymer. J. Mater. Chem. A 1 (2013) 11246-11260, DOI: 10.1039/c3ta12224g.

[43] Y. Liu, Z. Liu, Y. Liu, H. Hu, Y. Li, P. Yan, B. Yu, F. Zhou. One-step modification of fabrics with bioinspired polydopamine@octadecylamine nanocapsules for robust and healable self-cleaning performance. Small 11 (2014) 426-431, DOI: 10.1002/smll.201402383.

[44]A. Milionis, D. Fragouli, F. Brandi, I. Liakos, S. Barroso, R. Ruffilli, A. Athanassiou. Superhydrophobic/superoleophilic magnetic elastomers by laser ablation. Appl. Surf. Sci. 351 (2015) 7482, doi:10.1016/j.apsusc.2015.05.087.

[45]H. Blom, R. Yeh, R. Wojnarowski, M. Ling, Detection of degradation of ABS materials via DSC. J. Therm. Anal. Calorim. 83 (2006) 113-115, doi:10.1016/j.tca.2006.01.015.

[46] Y. Aoki, Rheological properties of abs polymer melts having a good dispersion of rubber particles. J. NonNewton. Fluid 22 (1986) 91-99, doi:10.1016/0377-0257(86)80005-2.

[47] M.H. Al-Saleh, H.K. Al-Anid, Y.A. Husain, M.E. El-Ghanem, S.A. Jawad, Impedance characteristics and conductivity of CNT/ABS nanocomposites. J. Phys. D: Appl. Phys. 46 (2013) 385305, doi:10.1088/00223727/46/38/385305.

[48] S.C. Chen, R.D. Chien, P.H. Lee, J.S. Huang, Effects of molding conditions on the electromagnetic interference performance of conductive ABS parts. J. Appl. Polym. Sci. 98 (2005) 1072-1080, DOI: 10.1002/app.22241.

[49] Y.S. Cho, S.H. Ahn, S.H. Lee, Fabrication and analysis of PMMA, ABS, PS, and PC superhydrophobic surfaces using the spray method. J. Korean Phys. Soc. 63 (2013) 218-224, DOI 10.3938/jkps.63.218.

[50]H. Jin, X. Tian, O. Ikkala, R.H.A. Ras, Preservation of superhydrophobic and superoleophobic properties upon wear damage. ACS Appl. Mater. Interfaces 5 (2013) 485-488, DOI: 10.1021/am302541f.

[51]B.J. Sparks, E F.T. Hoff, L. Xiong, J.T. Goetz, D.L. Patton, Superhydrophobic hybrid inorganic-organic thiol-ene surfaces fabricated via spray-deposition and photopolymerization. ACS Appl. Mater. Interfaces 5 (2013) 1811-1817, DOI: 10.1021/am303165e.

[52]T.M. Schutzius, I.S. Bayer, G.M. Jursich, A. Das, C.M. Megaridis, Superhydrophobic-superhydrophilic binary micropatterns by localized thermal treatment of Polyhedral Oligomeric Silsesquoixane (POSS)Silica films. Nanoscale 4 (2012) 5378-5385, doi: 10.1039/c2nr30979c. 
[53] A. Steele, I. Bayer, E. Loth, Inherently superoleophobic nanocomposite coatings by spray atomization. Nano Lett. 9 (2009) 501-505, DOI: 10.1021/nl8037272.

[54]I.S. Bayer, A. Steele, E. Loth, Superhydrophobic and electroconductive carbon nanotube-fluorinated acrylic copolymer nanocomposites from emulsions. Chem. Eng. J. 221 (2013) 522-530.

[55]X. Zhu, Z. Zhang, G. Ren, J. Yang, K. Wang, X. Xu, X. Men, X. Zhou, A novel superhydrophobic bulk material. J. Mater. Chem. 22 (2012) 20146-20148, DOI: 10.1039/C2JM33769J.

[56] X. Zhu, Z. Zhang, Y. Yang, X. Xu, X. Men, X. Zhou, Facile fabrication of a superhydrophobic fabric with mechanical stability and easy-repairability. J. Colloid Interf. Sci. 380 (2012) 182-186, doi: 10.1016/j.jcis.2012.04.063.

[57] S.A. Mahadik, D.B. Mahadik, M.S. Kavale, V.G. Parale, P.B. Wagh, H.C. Barshilia, S.C. Gupta, N.D. Hedge, A.V. Rao, Thermally stable and transparent superhydrophobic sol-gel coatings by spray method. J. Sol-Gel Sci. Technol. 63 (2012) 580-586, DOI 10.1007/s10971-012-2798-3.

[58] Y. Xiu, D.W. Hess, C.P. Wong, UV and thermally stable superhydrophobic coatings from sol-gel processing. J. Colloid Interf. Sci. 326 (2008) 465-470, doi:10.1016/j.jcis.2008.06.042.

[59]J. Lin, H. Chen, T. Fei, C. Liu, J. Zhang, Highly transparent and thermally stable superhydrophobic coatings from the deposition of silica aerogels. Appl. Surf. Sci. 273 (2013) 776-786.

[60] Budunoglu, H.; Yildirim, A.; Guler, M. O.; Bayindir, M. Highly Transparent, Flexible, and Thermally Stable Superhydrophobic ORMOSIL Aerogel Thin Films. ACS Appl. Mater. Interfaces 2011, 3, 539-545, doi:10.1016/j.apsusc.2013.02.134.

[61]H.S. Lim, J.H. Baek, K. Park, H.W. Shin, J. Kim, J.H. Cho, Multifunctional hybrid fabrics with thermally stable superhydrophobicity. Adv. Mater. 22 (2010) 2138-2141, DOI: 10.1002/adma.200903074.

[62] G. Gong, J. Wu, J. Liu, N. Sun, Y. Zhao, L. Jiang, Bio-inspired adhesive superhydrophobic polyimide mat with high thermal stability. J. Mater. Chem. 22 (2012) 8257-8262, DOI: 10.1039/C2JM16503A.

[63] Y.S. Ma, Y.J. Man, M. Shakerzadeh, H.L. Seet, R. Ji, R.Y. Zheng, H.J. Chung, X.Y. Chen, J.F. Hu, T. Yamamoto, R. Hempstead, Laser-heating-induced damage to ultrathin carbon overcoat in heat-assisted. Magnetic Recording. Tribol. Lett. 53 (2014) 303-310, DOI 10.1007/s11249-013-0268-x.

[64] S.Z. Shuja, B.S. Yilbas, Laser induced heating of coated carbon steel sheets: Consideration of melting and Marangoni flow. Opt. Laser Technol. 47 (2013) 47-55, doi:10.1016/j.optlastec.2012.07.029. 


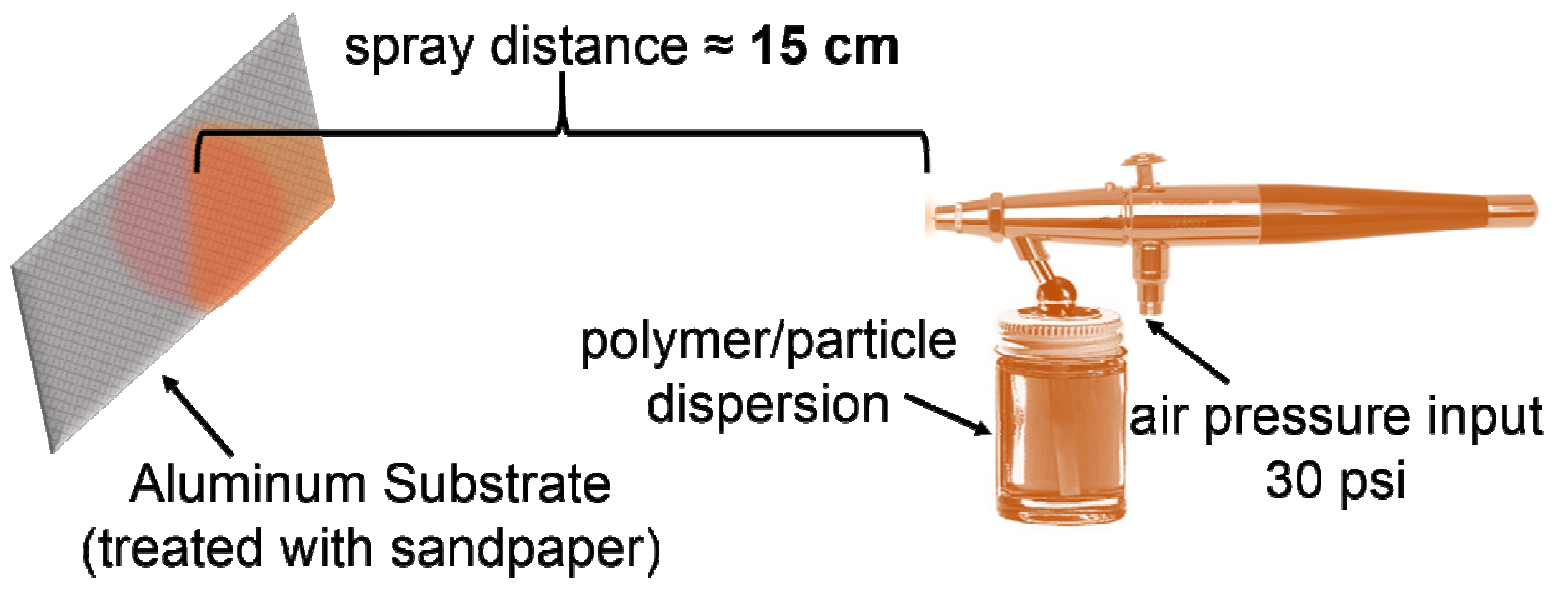

Fig. 1. Schematic of the experimental spray apparatus. 
Before thermal treatment
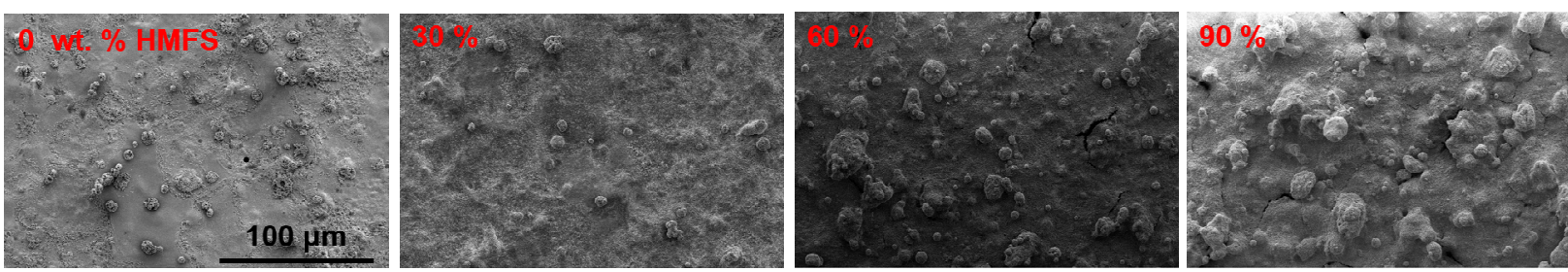

After thermal treatment at $240^{\circ} \mathrm{C}$ for $30 \mathrm{~min}$
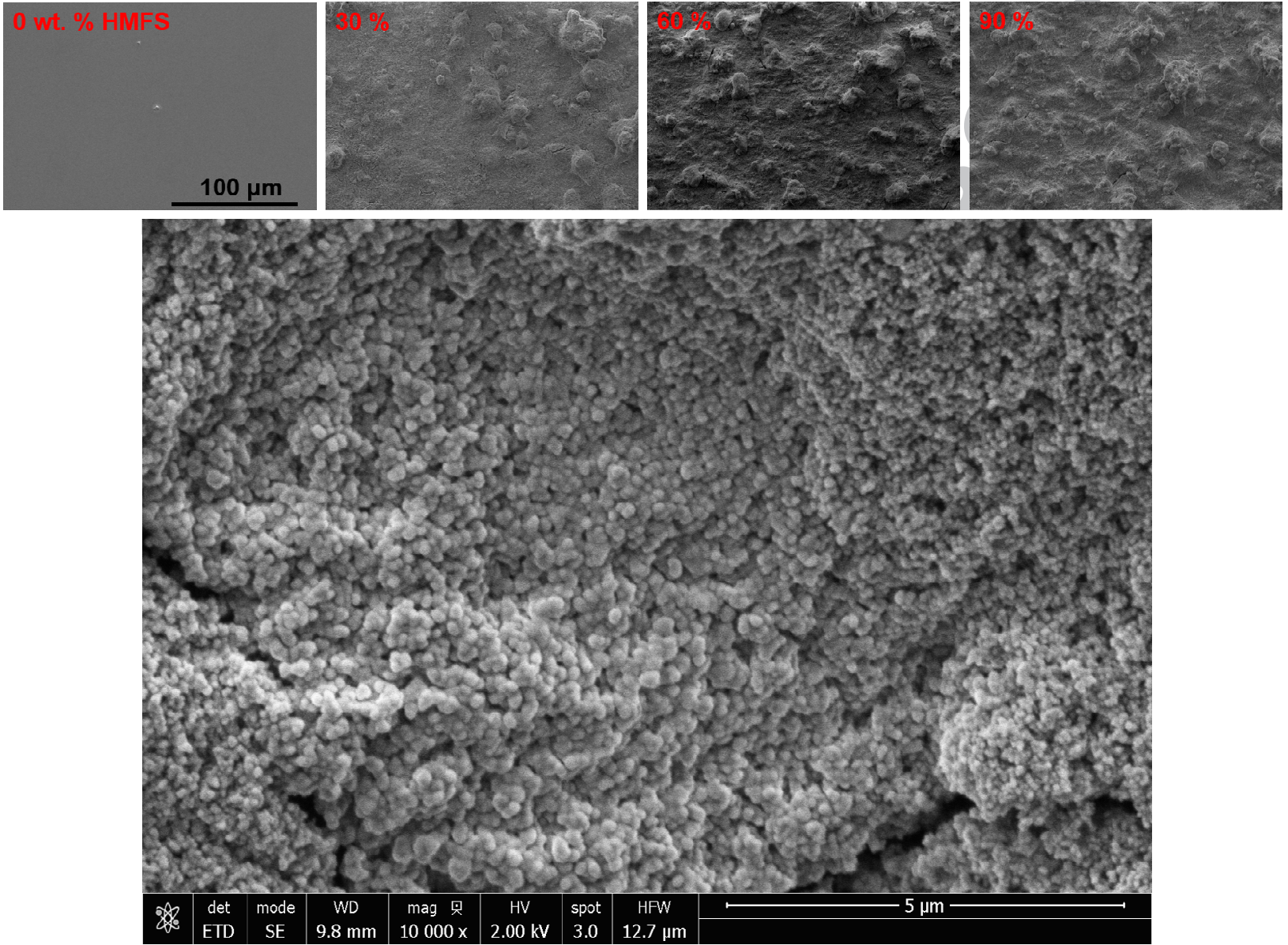

Fig. 2. Top panel: SEM images of the nanocomposite coatings for different loading in HMFS before and after the thermal treatment. Bottom panel: High magnification image of the surface structure of the superhydrophobic surface containing $30 \%$ silica nanoparticles. 

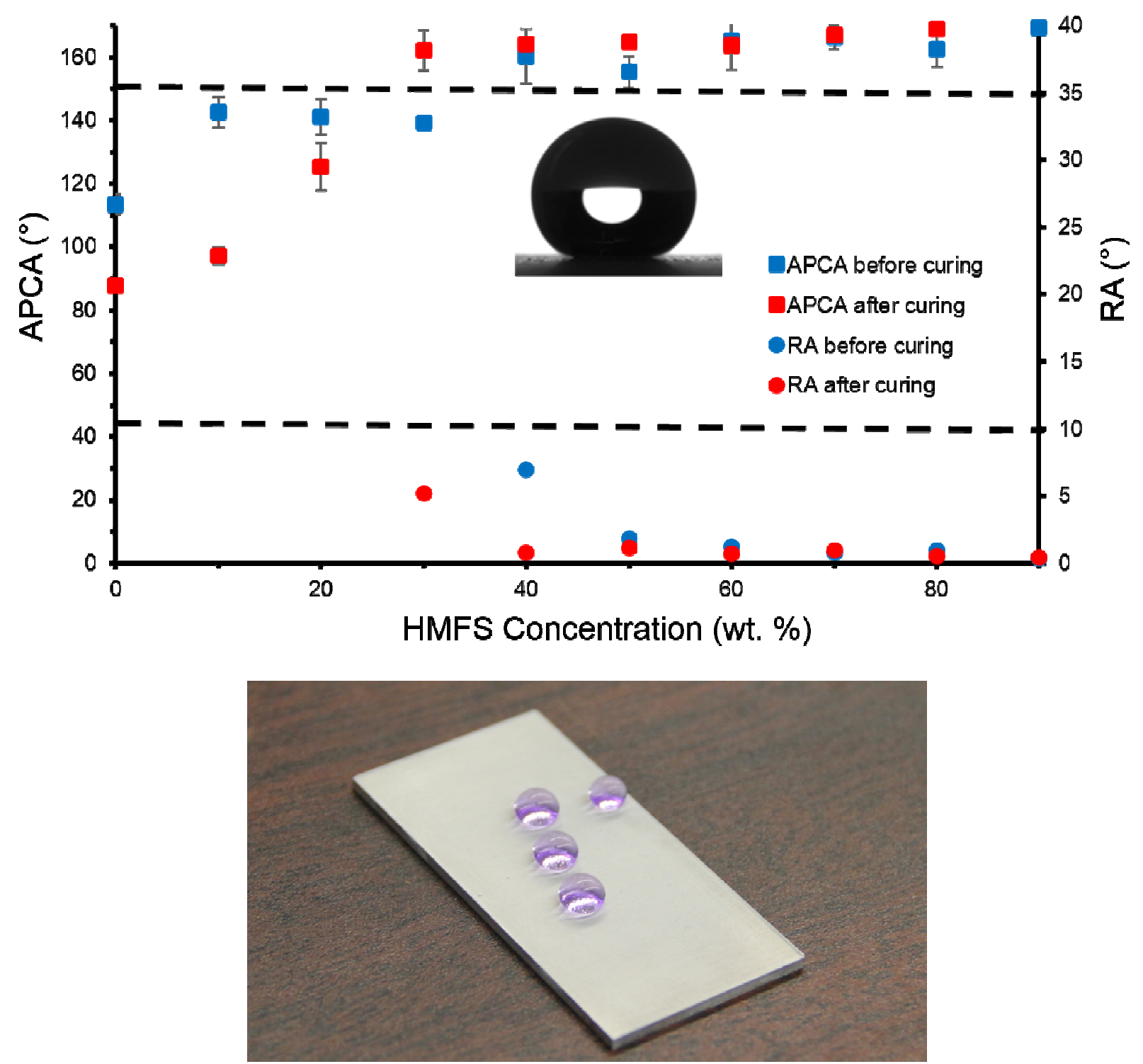

Fig. 3. Top panel: APCA and RA measurements plotted against the HMFS concentration before and after thermal curing. Bottom panel: Photograph of colored water droplets on the superhydrophobic ABS nanocomposite (40\% nanoparticles). 

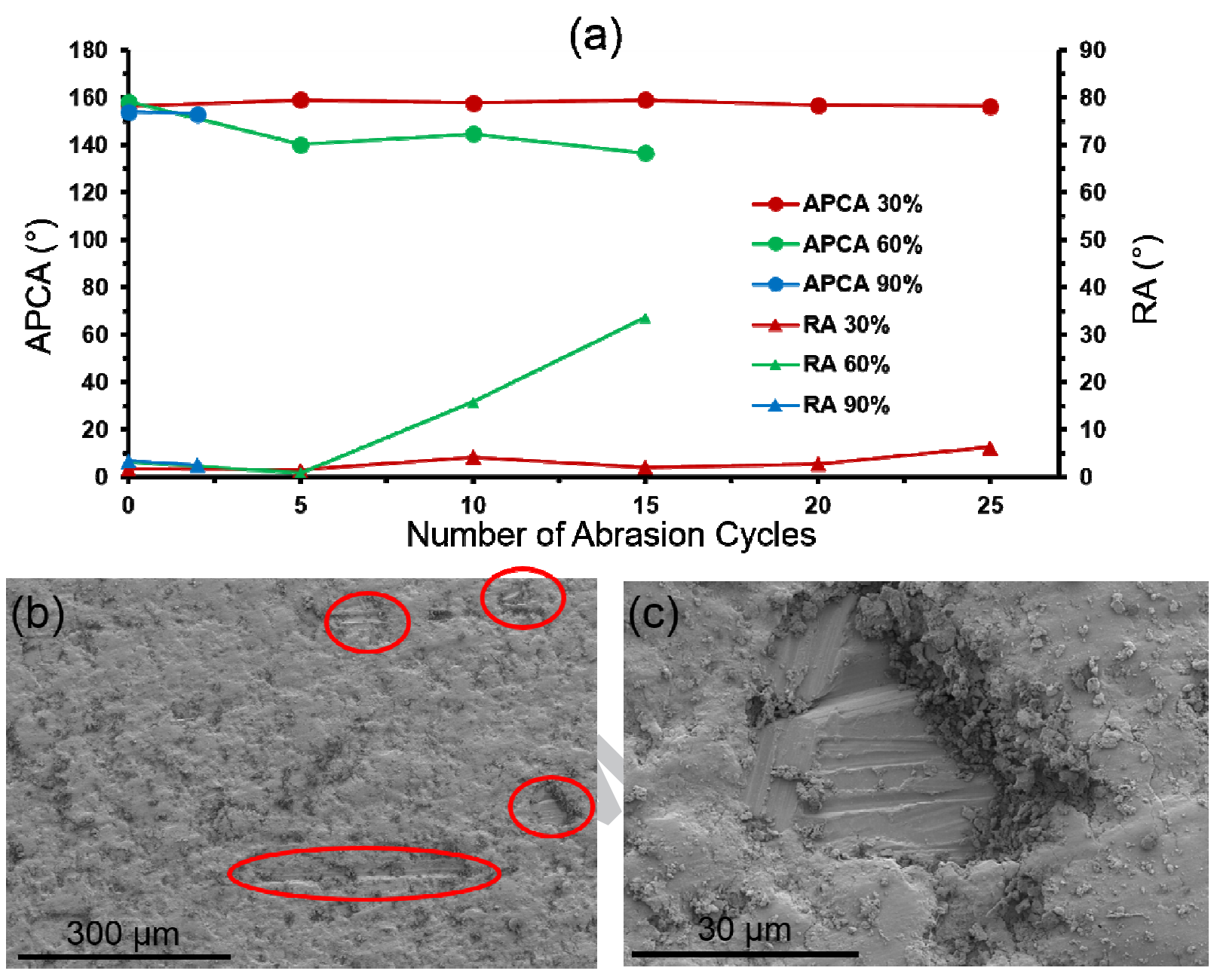

Fig. 4. (a) APCA and RA measurements plotted against the number of linear abrasion cycles. Three different nancomposites were tested with different HMFS concentration (30,60 and $90 \%$ by weight). (b) SEM image of the wear damaged areas on the surface marked by red circles. (c) High magnification SEM image showing the damaged area with exposed aluminum substrate. 

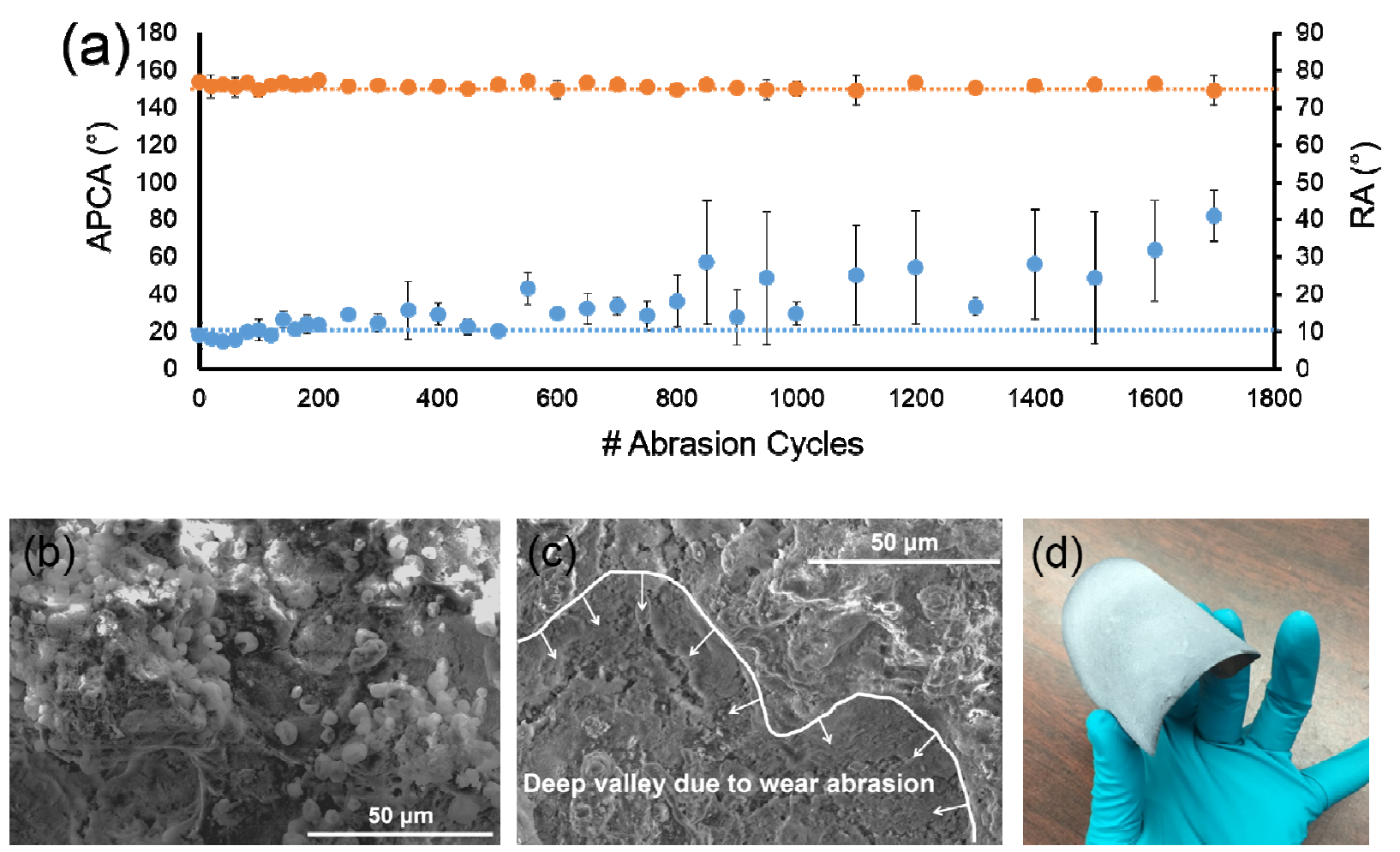

Fig. 5. (a) APCA and RA measurements plotted against the number of linear abrasion cycles. Bottom: SEM images of the as prepared (a) and abraded (b) areas of the coatings. (d) Photograph of the durable superhydrophobic nanocomposite applied on a flexible rubber substrate. 


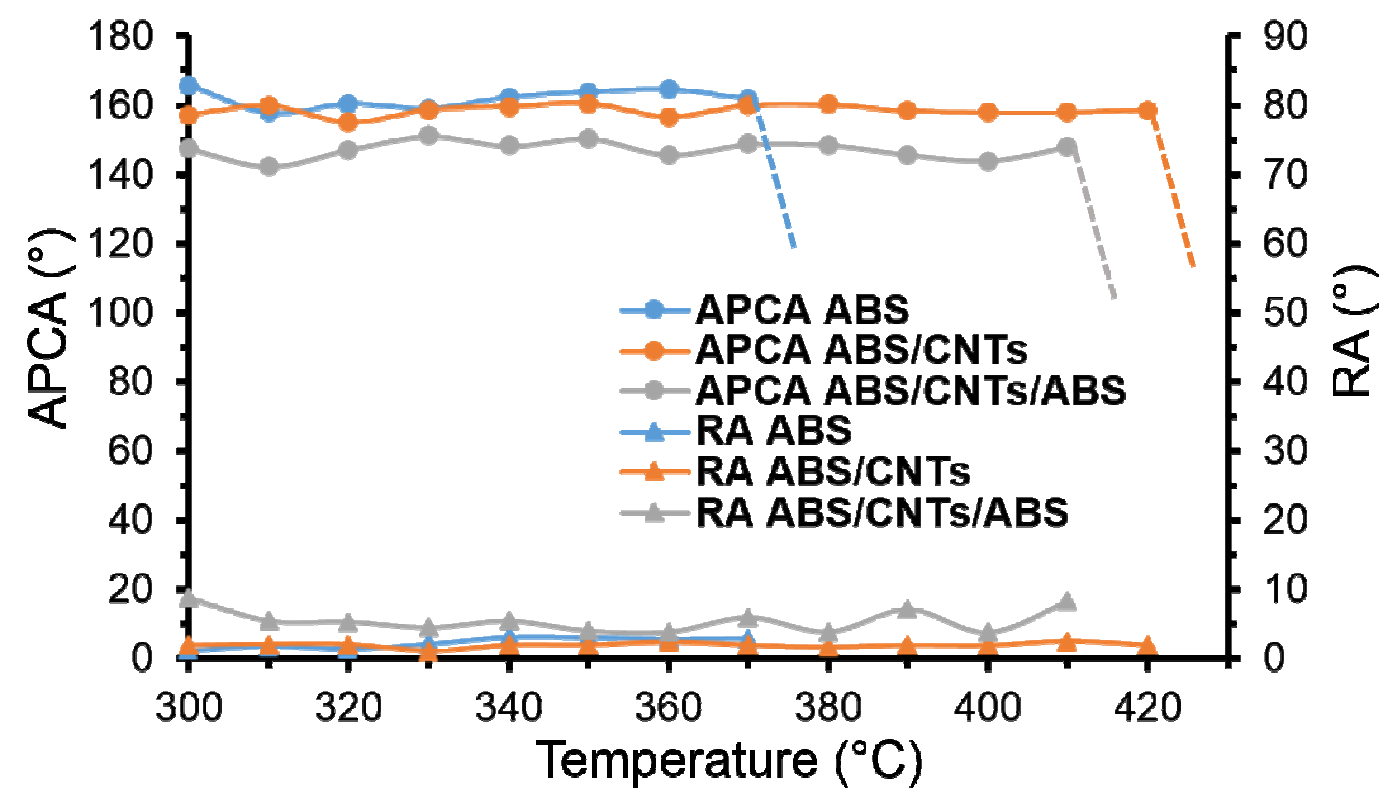

ABS ABS/CNTS

ABS/CNTs/ABS
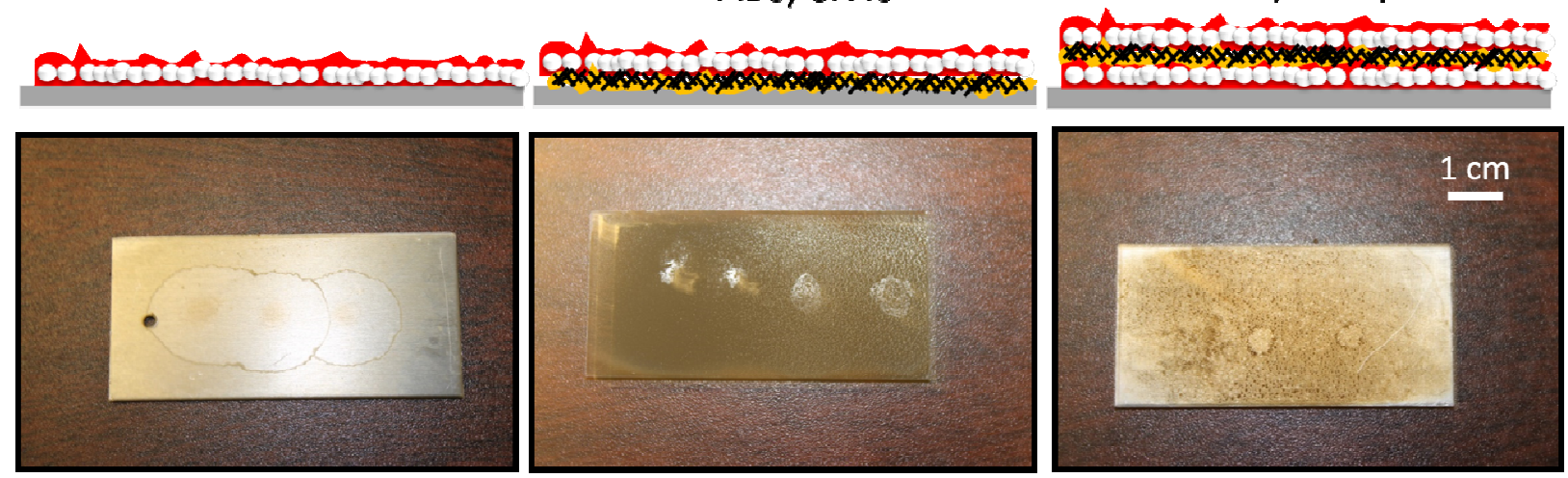

Fig. 6. Wetting properties plotted against the temperature for the three different samples. The schematics describe the coating layers and the photos below the samples when they lost their water repellence due to heat damage. 
Table 1. Surface roughness parameters of the nanocomposite coatings before and after the thermal treatment for different concentrations of HMFS.

\begin{tabular}{|c|c|c|c|c|}
\hline \multicolumn{5}{|c|}{ Before thermal treatment } \\
\hline $\begin{array}{c}\text { HMFS } \\
\%)\end{array}$ & Roughness $(\mu \mathrm{m})$ & $\begin{array}{c}\text { Root mean squared } \\
(\mu \mathrm{m})\end{array}$ & Kurtosis & Skewness \\
\hline 0 & $1.64 \pm 0.01$ & $2.04 \pm 0.05$ & $5.30 \pm 1.97$ & $1.64 \pm 0.01$ \\
\hline 30 & $2.10 \pm 0.12$ & $3.00 \pm 0.27$ & $11.04 \pm 2.96$ & $2.10 \pm 0.12$ \\
\hline 60 & $3.41 \pm 0.08$ & $4.65 \pm 0.22$ & $7.78 \pm 2.01$ & $2.05 \pm 0.33$ \\
\hline 90 & $4.44 \pm 0.21$ & $6.36 \pm 0.34$ & $8.18 \pm 0.72$ & $2.26 \pm 0.08$ \\
\hline
\end{tabular}

\begin{tabular}{|c|c|c|c|c|}
\hline \multicolumn{5}{|c|}{ After thermal treatment at $240^{\circ} \mathrm{C}$ for 30 min } \\
\hline $\begin{array}{c}\text { HMFS } \\
(\%)\end{array}$ & Roughness $(\mu \mathrm{m})$ & $\begin{array}{c}\text { Root mean squared } \\
(\mu \mathrm{m})\end{array}$ & Kurtosis & Skewness \\
\hline 0 & $0.28 \pm 0.09$ & $0.36 \pm 0.11$ & $3.69 \pm 1.31$ & $0.05 \pm 0.69$ \\
\hline 30 & $3.43 \pm 0.33$ & $4.73 \pm 0.45$ & $11.30 \pm 1.96$ & $2.40 \pm 1.07$ \\
\hline 60 & $3.15 \pm 0.14$ & $4.42 \pm 0.15$ & $9.14 \pm 1.21$ & $2.28 \pm 0.13$ \\
\hline 90 & $5.07 \pm 0.43$ & $7.43 \pm 0.55$ & $9.32 \pm 1.37$ & $2.48 \pm 0.18$ \\
\hline
\end{tabular}

